\title{
EDITORIAL
}

\section{T-cell interferon- $\gamma$ release assays: can we do better?}

\author{
A. Lalvani and K.A. Millington
}

$\triangle$ ccurate diagnosis of latent tuberculosis (TB) infection (LTBI) is scientifically challenging because of the low burden of dormant tubercle bacilli, which are not directly detectable or quantifiable. However, the strong cellular immune response triggered by LTBI serves as an amplified signal for the presence of these dormant bacilli. The first measure of the cellular immune response to be exploited as a marker of Mycobacterium tuberculosis infection, developed at the end of the 19th century, was the tuberculin skin test (TST), which measures a delayed type hypersensitivity response to tuberculin purified protein derivative. The development and validation of T-cell-based interferon (IFN)- $\gamma$ release assays (IGRAs) over the past decade represents a 100-yr upgrade in the diagnosis of LTBI [1,2]; the amplified signal measured is the ex vivo release of T-cell-derived IFN- $\gamma$. IGRAs' clearest advantage is increased specificity for detection of $M$. tuberculosis infection thanks to their utilisation of $M$. tuberculosis-specific antigens encoded in region of difference (RD)1, a genomic segment absent from the Bacille CalmetteGuérin (BCG) vaccine and most environmental mycobacteria.

Currently, there are two forms of IGRA: either IFN- $\gamma$ secretion is measured in whole-blood by ELISA or IFN- $\gamma$-secreting Tcells are directly enumerated by enzyme-linked immunospot (ELISpot) assay. The two assays are commercially licensed as QuantiFERON-TB Gold In-Tube (Cellestis Inc, Carnegie, Australia) and T-SPOT.TB (Oxford Immunotec Ltd, Abingdon, UK). Because these tests measure IFN- $\gamma$ released in response to the RD1-encoded antigens early secretory antigenic target (ESAT)-6 and culture filtrate protein (CFP)-10 (and TB7.7 (Rv2645) in QuantiFERON-TB Gold In-Tube), specificity in populations at very low risk for LTBI is higher than that of TST, approaching $100 \%[2,3]$. Because there is no gold standard test for LTBI, surrogate markers are used to estimate the diagnostic sensitivity of IGRAs. When using active TB as a surrogate for LTBI, the sensitivity of QuantiFERON-TB Gold In-Tube is 70\% (95\% confidence interval (CI) $63-78 \%)$ and of T-SPOT.TB is $90 \%$ (95\% CI $86-93 \%)$ [4]. The clinical utility of these tests hinges crucially on diagnostic sensitivity. In LTBI, sensitivity is especially important because targeted testing policies for LTBI are predicated on accurate diagnosis and

Tuberculosis Immunology Group, Dept of Respiratory Medicine, National Heart and Lung Institute, Imperial College London, London, UK.

STATEMENT OF INTEREST: Statements of interest for all authors of this manuscript can be found at www.erj.ersjournals.com/misc/statements.shtml

CORRESPONDENCE: A. Lalvani, Tuberculosis Immunology Group, Dept of Respiratory Medicine, National Heart and Lung Institute, Imperial College London, Norfolk Place, London, W2 1PG, UK. Fax: 44 2072628913. E-mail: a.lalvani@imperial.ac.uk preventive treatment of those at highest risk of developing active $\mathrm{TB}$, i.e. immunocompromised patients (e.g. HIV coinfected or iatrogenically immunosuppressed) and young children $[5,6]$. For evaluation of patients with suspected active $\mathrm{TB}$, tests of infection cannot be used to rule in TB disease as they cannot differentiate between active TB and LTBI. Rather, the predominant contribution of a test of infection is to enable clinicians rapidly to rule out a possible diagnosis of TB, which requires a test with very few false-negatives, i.e. high sensitivity. Although the diagnostic sensitivity of IGRAs is higher than that of the TST, their real-life clinical use demands higher sensitivity. So the key question is whether we can do better.

One approach that has been used for both ELISpot and ELISA to increase diagnostic sensitivity is incorporation of additional antigens of established high specificity [7]. In a recent large prospective study of patients with suspected $\mathrm{TB}$, incorporation of a novel RD-1-encoded antigen, RV3879c, alongside ESAT-6 and CFP-10 (ELISpot ${ }^{\text {PLUS }}$ ), significantly improved diagnostic sensitivity over the standard ELISpot test [8]. The addition of Rv2645 in QuantiFERON-TB Gold In-Tube significantly improved sensitivity of this assay in diagnosing active TB over QuantiFERON-TB Gold (which, like T-SPOT.TB, contains only ESAT-6 and CFP-10) without compromising specificity [9], in agreement with the observed higher positive results using QuantiFERON-TB Gold In-Tube than QuantiFERON-TB Gold in a cross-sectional study of healthy adults in South Africa [10].

Alternative readouts to measure IFN- $\gamma$ release by $M$. tuberculosis-specific T-cells have also been explored. For example, using flow cytometry, ESAT-6-specific IFN- $\gamma$ responses in peripheral blood mononuclear cells were detected in $13(87 \%)$ out of $15 \mathrm{~TB}$ patients [11] and measuring IFN- $\gamma$ mRNA from ESAT-6-stimulated peripheral blood mononuclear cells using quantitative PCR had a sensitivity of $65 \%$ in 54 active TB cases and LTBI [12].

Although IFN- $\gamma$ is a pivotal cytokine in the immune response to $M$. tuberculosis, are there alternative or additional cytokines that could be used to improve detection of $M$. tuberculosis infection? An essential role of IFN- $\gamma$ in host immunity against M. tuberculosis infection is activation of macrophages. Downstream chemokines induced by IFN- $\gamma$, namely IFN- $\gamma$ inducible protein 10 (IP-10 or CXCL10), monocyte chemoattractant protein (MCP)-2 and monokine-inducible protein (MIG or CXCL9) have been found in: delayed type hypersensitivity reactions to tuberculin purified protein derivative [13]; lymph node and lung ТВ granulomas; pleural effusions of TB patients [14]; and the plasma of TB patients [15]. 
These inflammatory chemokines, secreted by monocytes/ macrophages activated by pro-inflammatory cytokines or infection with a pathogen, traffic activated effector T-cells to inflamed foci. Because they are downstream and induced by IFN- $\gamma$, these chemokines may serve as a more amplified readout than IFN- $\gamma$ itself, thereby yielding higher sensitivity. IP-10 measured with a multiplex bead array identified four out of seven patients with active TB who had either negative or indeterminate QuantiFERON-TB Gold In-Tube results [16]. In another study, intracellular MIG, measured by flow cytometry, was higher in TB patients compared with BCG vaccinees [17].

In this issue of the European Respiratory Journal, RUHWALD et al. [18] evaluate whether measuring IP-10 and MCP-2 in addition to IFN- $\gamma$ secretion in response to $M$. tuberculosis-specific antigens enhances test sensitivity for diagnosis of active TB. In this proof-of-principle case-control study with 204 participants IFN- $\gamma$, IP-10 and MCP-2 released from whole blood stimulated with ESAT-6, CFP-10 and TB7.7 peptides for $18 \mathrm{~h}$ were measured using ELISA for IFN- $\gamma$ (QuantiFERON-TB Gold In-Tube) or multiplex bead arrays with flow cytometry for IP-10 and MCP-2. Overall, 5, 8 and 11\% of assays in TB patients were indeterminate for IFN- $\gamma$, IP-10 and MCP-2, respectively. None of the controls had indeterminate assays. Excluding indeterminate results, QuantiFERON-TB Gold InTube was positive in $86 \%$ out of 80 culture and/or PCRpositive TB patients, IP-10 was positive in $89 \%$ of patients and MCP-2 was positive in $80 \%$ of patients. Specificity of $100 \%$ with QuantiFERON-TB Gold In-Tube test in 124 controls with no risk factors for LTBI ( $31 \%$ of whom were BCG vaccinated) was reduced to 93 and 99\% when measuring IP-10 and MCP-2, respectively. Interestingly, decreasing the cut-off value for a positive QuantiFERON-TB Gold In-Tube result from the manufacturer's recommendations to $4 \mathrm{pg} \cdot \mathrm{mL}^{-1}$ increased the sensitivity of this test by $14 \%$ whilst maintaining high specificity in this population. In contrast, decreasing the cutoff value for a positive IP-10 result increased sensitivity accompanied by a decrease in specificity. Measuring IP-10 in addition to IFN- $\gamma$ significantly improved sensitivity by $4 \%$ over the QuantiFERON-TB Gold In-Tube test alone $(p<0.009)$ while only slightly compromising specificity, which decreased from 100 to $98 \%$. Measuring MCP-2 in addition to IFN- $\gamma$ did not significantly improve sensitivity. Importantly, immunosuppression was not significantly associated with negative or indeterminate results for any of the tests.

These results are promising for the combined readout of IFN- $\gamma /$ IP-10 as a potentially more sensitive marker of $M$. tuberculosis infection than current IGRAs. However, as with the clinical validation of IGRAs, the IFN- $\gamma /$ IP-10 test should now be prospectively evaluated in large numbers of unselected patients presenting with suspected active $\mathrm{TB}$ in routine practice to determine its true diagnostic accuracy and clinical utility, as was recently done for the next-generation ELISpot $^{\text {PLUS }}$ [8]. And ideally, such studies should include significant numbers of patients in whom TB is most difficult to diagnose, i.e. patients with HIV co-infection and children. Evaluating the performance of the IFN- $\gamma /$ IP-10 test in LTBI will require correlation of test results with epidemiologically well-defined exposure to TB, as was first carried out for IGRAs in 2001 [19]. Ultimately, the prognostic value of the IFN- $\gamma /$ IP10 test for subsequent development of active TB will need to be established to confirm its validity as a test of LTBI, as was recently done for QuantiFERON-TB Gold In-Tube [20] and ELISpot [21].

Given the complexity of the cellular immune response to $M$. tuberculosis infection, can we infer additional useful clinical and biological information about our patients by measuring other immunological mediators? Simultaneous measurement of interleukin (IL)-2 with IFN- $\gamma$ at the single T-cell level reveals T-cell cytokine profiles (defined as the relative proportions of antigen-specific T-cells secreting IFN- $\gamma$-only, IFN- $\gamma / \mathrm{IL}-2$ or IL-2-only) that correlate with pathogen and antigen load across a range of viral infections [22]. These functional cytokine profiles also correlate with bacterial and antigen load in TB, shifting dynamically in response to successful treatment [23], suggesting a potentially clinically useful role for this new tool for TB treatment monitoring and test of cure [24]. Furthermore, measuring mRNA expression of several cytokines in response to ESAT-6 stimulation revealed that for a combination of IL-8, FOXP3 and IL-12 $\beta$, signatures that discriminated active TB from LTBI could be defined [12]. Whether detection of T-cell responses to specific antigens or peptide epitopes can discriminate active from latent $\mathrm{TB}$ is also being actively investigated [25-27].

Thus, current interferon- $\gamma$ release assays, when viewed in the context of advances in T-cell immunology over the past decade, are in reality an evolving work in progress that embody the state of our knowledge at the end of the 20th century [1]. The study by RuHWALD et al. [18] provides a cogent example of taking more recent discoveries about the immune response to tuberculosis and translating them into proof-ofprinciple human studies in order to establish their potential clinical relevance. It is now widely recognised that interferon- $\gamma$ release assays were the 100-yr upgrade on tuberculin skin test for diagnosis of latent tuberculosis infection $[1,2,4,6]$. What studies such as those by RUHWALD et al. [18] show us is that the next generation of immune-based diagnostic tests for tuberculosis is not another century away but more likely just around the corner.

\section{REFERENCES}

1 Barnes PF. Diagnosing latent tuberculosis infection: the 100-year upgrade. Am J Respir Crit Care Med 2001; 163: 807-808.

2 Lalvani A. Diagnosing tuberculosis infection in the 21st century: new tools to tackle an old enemy. Chest 2007; 131: 1898-1906.

3 Richeldi L. An update on the diagnosis of tuberculosis infection. Am J Respir Crit Care Med 2006; 174: 736-742.

4 Pai M, Zwerling A, Menzies D. Systematic review: T-cellbased assays for the diagnosis of latent tuberculosis infection: an update. Ann Intern Med 2008; 149: 177-184.

5 American Thoracic Society. Targeted tuberculin testing and treatment of latent tuberculosis infection. Am J Respir Crit Care Med 2000; 161: S221-S247.

6 National Collaborating Centre for Chronic Conditions. Tuberculosis: Clinical Diagnosis and Management of Tuberculosis, and Measures for its Prevention and Control. London, Royal College of Physicians, 2006. 
7 Liu XQ, Dosanjh D, Varia H, et al. Evaluation of T-cell responses to novel RD1- and RD2-encoded Mycobacterium tuberculosis gene products for specific detection of human tuberculosis infection. Infect Immun 2004; 72: 2574-2581.

8 Dosanjh DP, Hinks TS, Innes JA, et al. Improved diagnostic evaluation of suspected tuberculosis. Ann Intern Med 2008; 148: 325-336.

9 Harada N, Higuchi K, Yoshiyama T, et al. Comparison of the sensitivity and specificity of two whole blood interferon-gamma assays for $M$. tuberculosis infection. J Infect 2008; 56: 348-353.

10 Mahomed H, Hughes EJ, Hawkridge T, et al. Comparison of mantoux skin test with three generations of a whole blood IFN-gamma assay for tuberculosis infection. Int $J$ Tuberc Lung Dis 2006; 10: 310-316.

11 Tesfa L, Koch FW, Pankow W, Volk HD, Kern F. Confirmation of Mycobacterium tuberculosis infection by flow cytometry after ex vivo incubation of peripheral blood $\mathrm{T}$ cells with an ESAT-6-derived peptide pool. Cytometry 2004; 60: 47-53.

$12 \mathrm{Wu}$ B, Huang C, Kato-Maeda M, et al. Messenger RNA expression of IL-8, FOXP3, and IL-12 $\beta$ differentiates latent tuberculosis infection from disease. J Immunol 2007; 178: 3688-3694.

13 Kaplan G, Luster AD, Hancock G, Cohn ZA. The expression of a gamma interferon-induced protein (IP-10) in delayed immune responses in human skin. J Exp Med 1987; 166: 1098-1108.

14 Okamoto M, Kawabe T, Iwasaki Y, et al. Evaluation of interferon-gamma, interferon-gamma-inducing cytokines, and interferon-gamma-inducible chemokines in tuberculous pleural effusions. J Lab Clin Med 2005; 145: 88-93.

15 Juffermans NP, Verbon A, van Deventer SJ, et al. Elevated chemokine concentrations in sera of human immunodeficiency virus (HIV)-seropositive and HIV-seronegative patients with tuberculosis: a possible role for mycobacterial lipoarabinomannan. Infect Immun 1999; 67: 4295-4297.

16 Ruhwald M, Bjerregaard-Andersen M, Rabna P, Kofoed K, Eugen-Olsen J, Ravn P. CXCL10/IP-10 release is induced by incubation of whole blood from tuberculosis patients with ESAT-6, CFP10 and TB7.7. Microbes Infect 2007; 9: 806-812.

17 Abramo C, Meijgaarden KE, Garcia D, et al. Monokine induced by interferon gamma and IFN-gamma response to a fusion protein of Mycobacterium tuberculosis ESAT- 6 and CFP-10 in Brazilian tuberculosis patients. Microbes Infect 2006; 8: 45-51.

18 Ruhwald M, Bodmer T, Maier C, et al. Evaluating the potential of IP-10 and MCP-2 as biomarkers for the diagnosis of tuberculosis. Eur Respir J 2008; 32: 1607-1615.

19 Lalvani A, Pathan AA, Durkan H, et al. Enhanced contact tracing and spatial tracking of Mycobacterium tuberculosis infection by enumeration of antigen-specific T cells. Lancet 2001; 357: 2017-2021.

20 Diel R, Loddenkemper R, Meywald-Walter K, Niemann S, Nienhaus A. Predictive value of a whole-blood IFN- $\gamma$ assay for the development of active TB disease. Am J Respir Crit Care Med 2008; 177: 1164-1170.

21 Bakir M, Millington KA, Soysal A, et al. Prognostic value of a $\mathrm{T}$ cell based interferon-gamma biomarker in child tuberculosis contacts. Ann Intern Med 2008; (in press).

22 Pantaleo G, Harari A. Functional signatures in antiviral Tcell immunity for monitoring virus-associated diseases. Nat Rev 2006; 6: 417-423.

23 Millington KA, Innes JA, Hackforth S, et al. Dynamic relationship between IFN-gamma and IL-2 profile of Mycobacterium tuberculosis-specific T cells and antigen load. J Immunol 2007; 178: 5217-5226.

24 Lalvani A, Millington KA. T cells and tuberculosis: beyond interferon-gamma. J Infect Dis 2008; 197: 941-943.

25 Hougardy JM, Schepers K, Place S, et al. Heparin-bindinghemagglutinin-induced IFN-gamma release as a diagnostic tool for latent tuberculosis. PLoS One 2007; 2: e926.

26 Vincenti D, Carrara S, De Mori P, et al. Identification of early secretory antigen target- 6 epitopes for the immunodiagnosis of active tuberculosis. Mol Med 2003; 9: 105-111.

27 Leyten EM, Lin MY, Franken KL, et al. Human T-cell responses to 25 novel antigens encoded by genes of the dormancy region of Mycobacterium tuberculosis. Microbes Infect 2006; 8: 2052-2060. 